\title{
Unusually High Number of Eschars on the Face of a Patient with Scrub Typhus
}

In fall 2010, a 63-year-old man was admitted to Wonkwang University Hospital with a 1-week history of fever and chills, and a rash over his entire body. He was fond of mountain climbing and occasionally rested on the ground while climbing mountains. At the time of admission, he appeared acutely ill, with a body temperature of $38.6^{\circ} \mathrm{C}$. On physical examination, the cervical lymph nodes were tender. The patient had two different types of skin rash: erythematous maculopapules over the entire body except the face and painless erythematous, crusted papules on the face (Fig. 1). He was suspected of having scrub typhus, and empirical antibiotic treatment with doxycycline was initiated. After 2 days of treatment, his symptoms began to improve. On the $5^{\text {th }}$ day, the rash on the face became the typical eschars (Fig. 2). The antibody titer against Orientia tsutsugamushi, detected using an indirect immunofluorescent antibody assay, was 1:2560. After 2 weeks of treatment, the eschars fell off, but postinflammatory hyperpigmentation remained. After 45 days, the patient's skin lesions had disappeared .

A previous study reported that eschars occurred in 4692\% of scrub typhus patients in Korea. Moreover, almost all of the patients had the typical single eschar, which was usually found on the chest above the umbilicus. We encountered a patient with scrub typhus who had an unusually large number of eschars on the face.

\section{Conflict of interest}

No potential conflict of interest relevant to this article was reported.

\section{In Tae Hwang and Jae Hoon Lee}

Department of Internal Medicine, Wonkwang University College of Medicine, Iksan, Korea

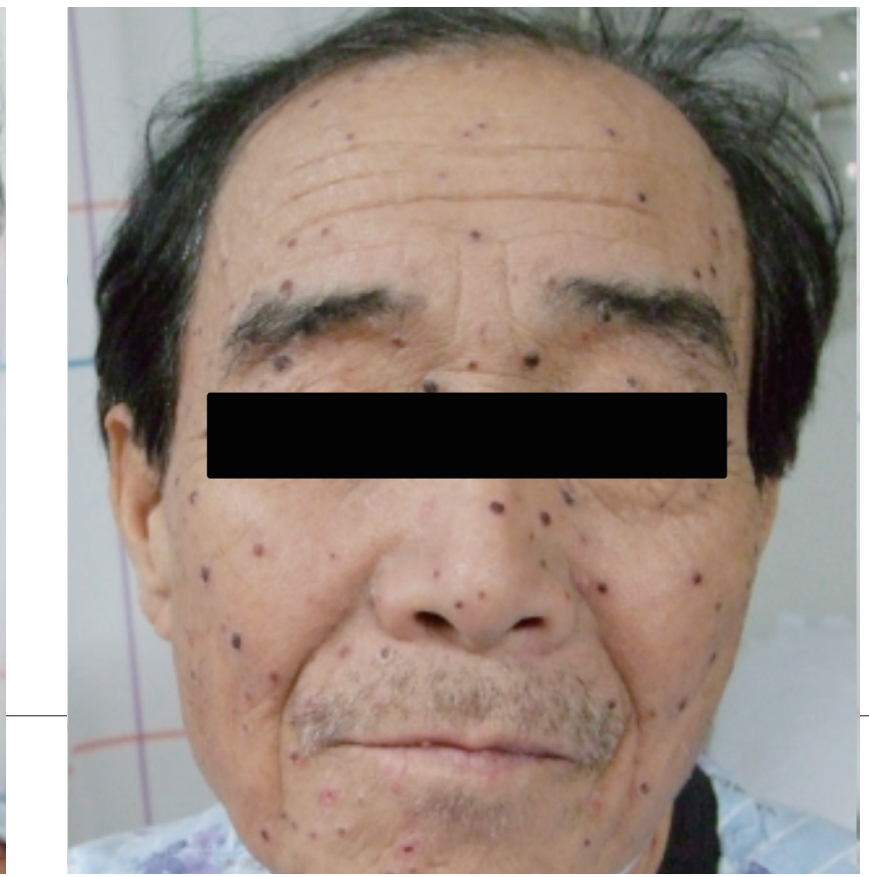

Figure 2. The papules became typical eschars on the $5^{\text {th }}$ day after admission.
Figure 1. Numerous erythematous crusted papules are observed on the face.

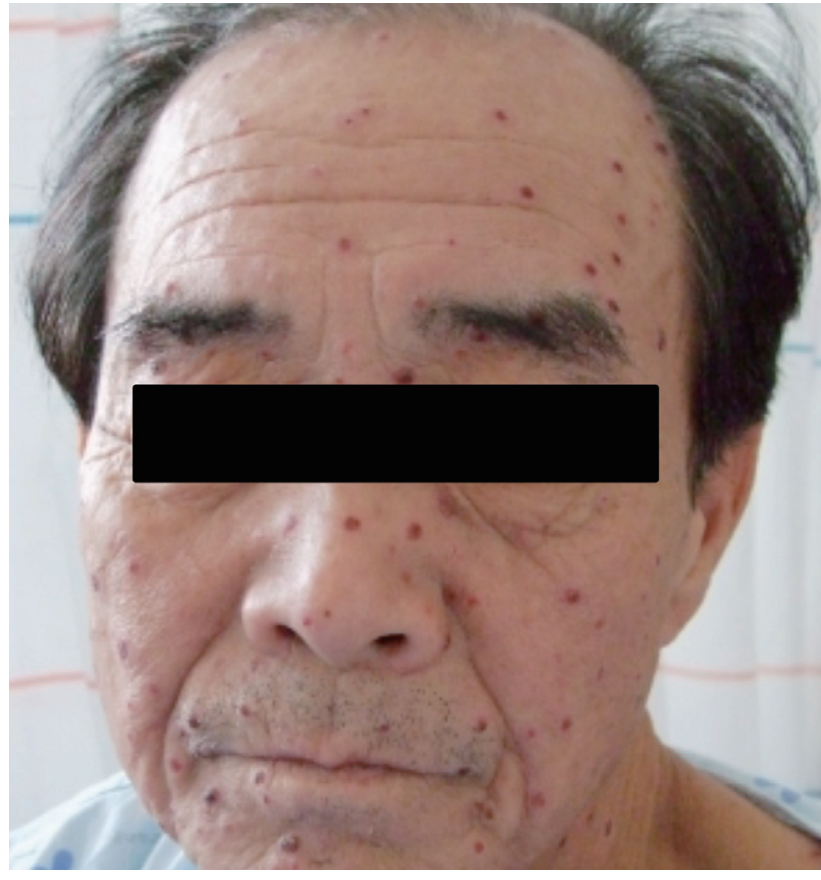

